\title{
Strategy for Designing Digital Filters for Embedded Platforms
}

\author{
Holman Montiel $^{\# 1}$, Edwar Jacinto ${ }^{\# 2}$, Fernando Martinez ${ }^{\# 3}$ \\ \# Technological Faculty,Universidad Distrital Francisco José de Caldas \\ Cll 68 D Bis A Sur No. 49F - 70, Bogotá D.C., Colombia \\ ${ }^{1}$ hmontiela@udistrital.edu.co, ${ }^{2}$ ejacintog@udistrital.edu.co \\ 3 fmartinezs@udistrital.edu.co
}

\begin{abstract}
This research focuses on the use of a methodological strategy for the implementation of digital filters on embedded systems. The purpose of the document is to perform a results analysis for the implementation of a FIR filter on a PSOC 5LP, comparing the proposed strategy with the actual response of the architecture used by the selected platform. In the document it will be shown the block diagram of the proposed solution, configuration parameters, algorithmic structures used, computational tools used to calculate operating parameters and the development of validation tests for the proposed strategy.
\end{abstract}

Keyword- Digital filters, PSOC, FIR, embedded systems.

\section{INTRODUCTION}

The growing development of electronic components of small size, has allowed to build high quality electronic prototypes at low cost. In particular, prototypes that incorporate a digital processing system, have the ability to execute tasks recorded in their memory. This characteristic makes them a central controller of certain embedded systems, which gives them a functional advantage over conventional digital circuits, since they adapt to different types of applications with a single processing system [1-3].

The versatility of this type of controller has allowed its manufacture by several manufacturers, among which stand out the family of programmable controllers (PSOC: Programmable System on Chip) developed by Cypress in 2002. The PSOC are integrated circuits of programmable matrix, that is, the peripherals are defined by sets of functions and assigned to any terminal of the integrated circuit [2-3].

The manufacture of PSOC and other digital systems allows the processing of large volumes of information and signals in hardware digitally, although the speed of response in these applications is reduced to the function of the scheduled tasks and the architecture of the controller. The applications in the areas associated to the digital processing of the signals have had great advances, some representative examples are: facial or voice recognition [4-5], the development of intelligent sensors [6], the manufacture of wireless audio transmitters [7], the design of biometric systems [8], among others.

One limitation that can occur during the implementation of this type of applications, is the increase in the margin of error between the measured value and the real value, since the signals that reach a controller can change its magnitude with a case of an external client, for example: the environmental conditions. This limitation has been partly solved thanks to the development of filters, which have the function of eliminating the effect caused by the factors [9-10].

In electronics, two types of filters are found, analog and digital. Analog filters are usually built as passive components such as resistors, capacitors and coils. Digital filters are constructed from algorithms that adapt to different processing systems, to improve the result obtained when interpreting a digital signal, for example, to eliminate the noise present in an audio signal reproduced by a mini-component.

The importance and characteristics of digital filters are presented in this paper, which is structured as follows: Section 1 presents the main characteristics of a digital filter. In section 2, a methodology for the design of a digital filter is taught analytically. Section 3 shows a way to implement a digital filter in an embedded system and the results obtained when comparing the proposed strategy versus the response of the embedded system.

\section{METHODOLOGY}

\section{A. Definition of Filter and Its Classification}

The word filter is used to denote a system that is responsible for excluding certain attributes of the object that is processing, for example, drinking water purifiers that are used to eliminate microorganisms harmful to health. More specifically in signal processing, filters are used to set a group of observable parameters of a signal eliminating the rest, including frequency [11].

The parameters of the filters designed to modify their output as a function of frequency are: the cutoff frequency, the step frequency and the attenuation level. The cutoff frequency sets the level below which the filter output is attenuated. The step frequency is the set of values that the filter output has in a given frequency 
range. The attenuation level is a factor that indicates how much the amplitude of the original signal is reduced at the filter output, normally expressed in $\mathrm{dB}$ (a decibel is used to express the power level of a signal). When a filter is constructed taking into account its domain in the frequency it is classified as follows [14-17]:

- Low-pass filter: This type of filter attenuates the signals with high frequencies and lets the low frequency signals pass (related to a given cutoff frequency).

- High-pass filter: This type of filter works in the opposite way to the low pass filter, since it attenuates the low frequency signals and lets the high frequency signals pass.

- Band-pass filter: This type of filter combines the characteristics of the aforementioned filters, since it lets signals pass with frequencies in a certain range.

- Eliminated band filter: This type of filter is the inverse of the band pass filter, since it eliminates signals with frequencies that are in a certain interval.

As mentioned before the filters can be classified as analog or digital. Analog filters are used to process signals that are in the continuous time domain and their complement are digital filters, since they process the signals that have a domain in discrete time. Discrete time signals are ideal for digital controllers, because they do not process signals in continuous time [14-17].

Digital controllers determine the magnitude of a signal in different ways. However, the fidelity of the processed signal regarding the original depends on the sampling time used to capture the information. The sampling frequency is the system's ability to sample a continuous time signal for one unit of time. For example, the sampling frequency used by a analog to digital converter (ADC) to digitize a voice signal is normally 44.1 $\mathrm{kHz}$, since the human voice is below this value (less than $20 \mathrm{kHz}$ ).

The sampled signals are filtered in a digital system using various filter types, of which the two most commonly used are mentioned:

IIR filter: The Infinite Impulse Response filter (IIR) is a type of filter that by inducing the system into an input with a unit impulse form, said system will not be at rest again. The output of the filter depends on the previous and present values in the input, which are temporarily stored in the memory of the controller. This type of filter is represented by the mathematical expressions of equations 1 and 2 [13].

$$
\begin{aligned}
& y[n]=\sum_{i=0}^{P} b_{i} x[n-i]-\sum_{j=1}^{Q} a_{i} y[n-i] \\
& H(z)=\frac{\sum_{i=0}^{P} b_{i} z^{-i}}{\sum_{j=0}^{Q} a_{i} z^{-j}}
\end{aligned}
$$

In the expressions $P$ and $Q$ determine the filter order, $a$ and $b$ determine the filter coefficients, an approximate model of the system is represented by the transfer function $H$ (z) [13].

FIR filter: The finite impulse response filter (FIR) has a response limited by a finite number of terms, which is estimated from the current and previous input values, because they depend on the input they are only known as non-recursive filters and their output is represented by the mathematical expression of equation 3 [11].

$$
y[n]=\sum_{k=0}^{L-1} h[k] * x[n-k]
$$

Where $h[k]$ is the filter transfer function, $x$ is the input value and $L$ determines the order of the filter. This relationship can also be represented by a transfer function as shown in equation 4 .

$$
H(z)=\sum_{k=0}^{L-1} h_{k} z^{-k}=h_{0}+h_{1} z^{-1}+h_{2} z^{-2} \ldots \ldots+h_{k-1} z^{-(L-1)}
$$

Some differences between the two filter types are: The difficulty in the design, since the FIR filters are usually designed by iterative methods and the IIR by means of transformations of numerical expressions or mathematical equations. Efficiency, FIR filters usually require less time to estimate the output than type IIR, since FIR type filters are not recursive, that is, they do not need feedback from the output, even though FIR filters require a greater memory space for its implementation.

However, these characteristics vary depending on the methodology used to design the filter, in particular to design a FIR filter, 3 methods are usually used: The method of the window or Fourier transform, the method of sampling in frequency and the method of optimal approximations. Of which the transformation method will be analyzed, since it allows an approximate model of the transfer function to be obtained analytically [11].

\section{DESIGN AND IMPLEMENTATION}

An approximate filter model is achieved by applying the window method, which consists of finding the appropriate coefficients of a FIR of order L, making a convolution between the transfer function that characterizes the ideal filter by different values of a function called a window [12].

To determine the function that characterizes the filter, it is assumed that $H\left(e^{j \omega}\right)$ is the Fourier transform in discrete time (from English, Discrete-time Fourier transform) of $h[n]$, as shown in equations 5 and 6 [11].

$$
H\left(e^{j \omega}\right)=\sum_{-\infty}^{\infty} h[n] e^{-j \omega n}
$$




$$
h[n]=\frac{1}{2 \pi} \int_{-\infty}^{\infty} H\left(e^{j \omega}\right) e^{j \omega n} d w
$$

This methodology is applied in the filters that work as a function of the frequency, in this example we have a low pass filter, which is established with a cutoff frequency $w_{0}$ as shown in equation 8 represented graphically in Fig.1. The negative component observed is due to the fact that the Fourier transform is symmetric around 0 , however, in practice the frequencies are always assumed to be positive.

$$
H\left(e^{j \omega}\right)=\left\{\begin{array}{ll}
1 & |w| \leq w_{0} \\
0 & |w|>w_{0}
\end{array}\right\}
$$
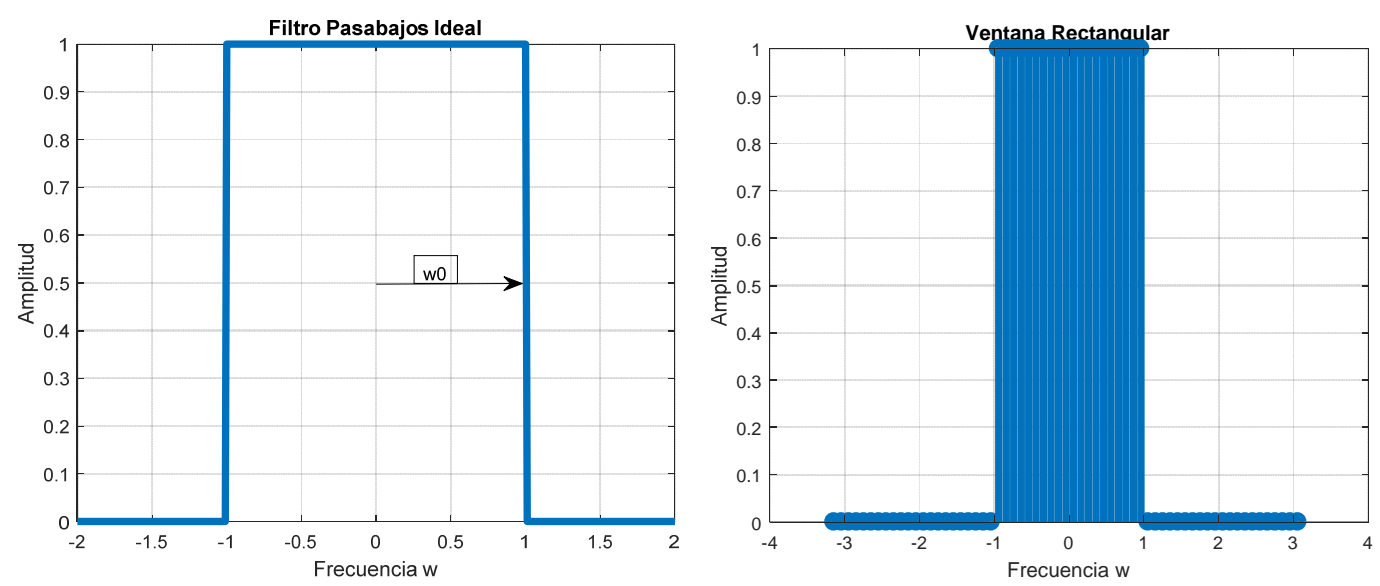
a)
Answer of $H\left(e^{j \omega}\right)$
b) Rectangular window $w$

Fig. 1. Model of the filter to be designed

When applying the Fourier transform to the approximate filter model, the result shown in equation 12 is obtained and a response is obtained as shown in Fig. 2a.

$$
\begin{aligned}
& h[n]=\frac{1}{2 \pi} \int_{-\infty}^{\infty} H\left(e^{j \omega}\right) e^{j \omega n} d w \\
& h[n]=\frac{1}{2 \pi} \int_{-w_{0}}^{w_{0}} 1 * e^{j \omega n} d w=\frac{1}{2 \pi}\left[\frac{1}{j n} e^{j \omega n}\right]_{-w_{0}}^{w_{0}}=\frac{1}{2 \pi}\left[\frac{1}{j n} e^{j \omega_{0} n}-\frac{1}{j n} e^{-j \omega_{0} n}\right] \\
& h[n]=\frac{1}{2 \pi}\left[\frac{1}{j n} e^{j \omega_{0} n}-\frac{1}{j n} e^{-j \omega_{0} n}\right] \\
& h[n]=\frac{1}{2 \pi}\left\{\left[\frac{1}{j n}\left(\cos \left(\omega_{0} n\right)+j \operatorname{sen}\left(\omega_{0} n\right)\right)\right]-\left[\frac{1}{j n}\left(\cos \left(\omega_{0} n\right)-j \operatorname{sen}\left(\omega_{0} n\right)\right)\right]\right\} \\
& h[n]=\frac{1}{2 \pi}\left(\frac{2 \operatorname{sen}\left(\omega_{0} n\right)}{n}\right)=\frac{\operatorname{sen}\left(\omega_{0} n\right)}{n \pi}
\end{aligned}
$$

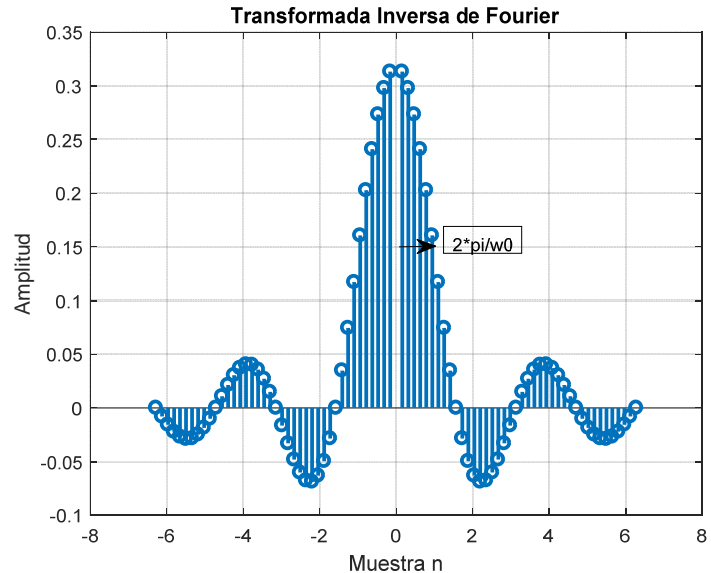

a) Answer of $h[n]$

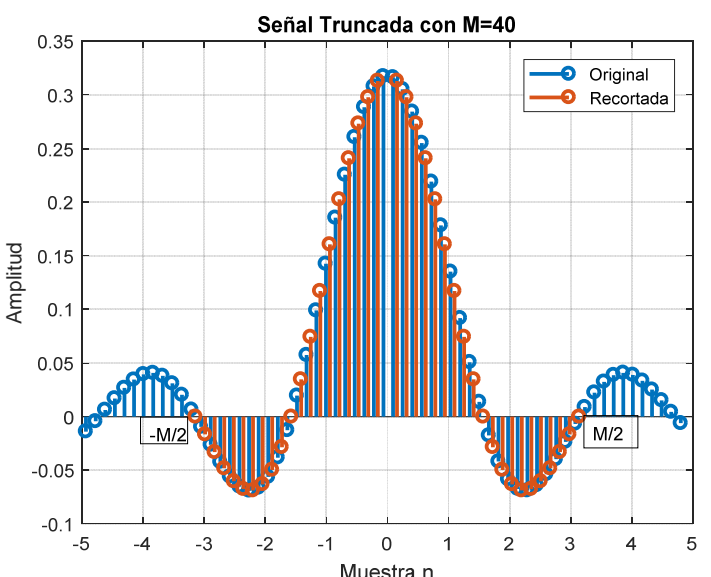

b) Answer of $h_{1}[n]$

Fig. 2. Behavior of the Fourier transform.

As indicated in the red box of Fig. 2a, the harmonics of the transformation reach small amplitudes, so much so that the result can be truncated around the central value, for which a set of values is fixed as observed in the rectangular window of the Figure $1 \mathrm{~b}$. When performing a multiplication between the functions, it is obtained a finite function $h_{1}[n]$ of rank M. 
One way to estimate the correct value of $\mathrm{M}$ is presented in equation 13 , however, this can be adjusted to reduce the mean square error (equation 14), which is calculated from the Parseval relation. The error determines the behavior of the filter output as shown in Fig. 3, the response of when $M$ tends to infinity (Blue Line) is stable and for different values these become unstable (Red Line $M=40$ ) or do not attenuate enough from the cutoff frequency (Yellow Line $\mathrm{M}=25$ ).

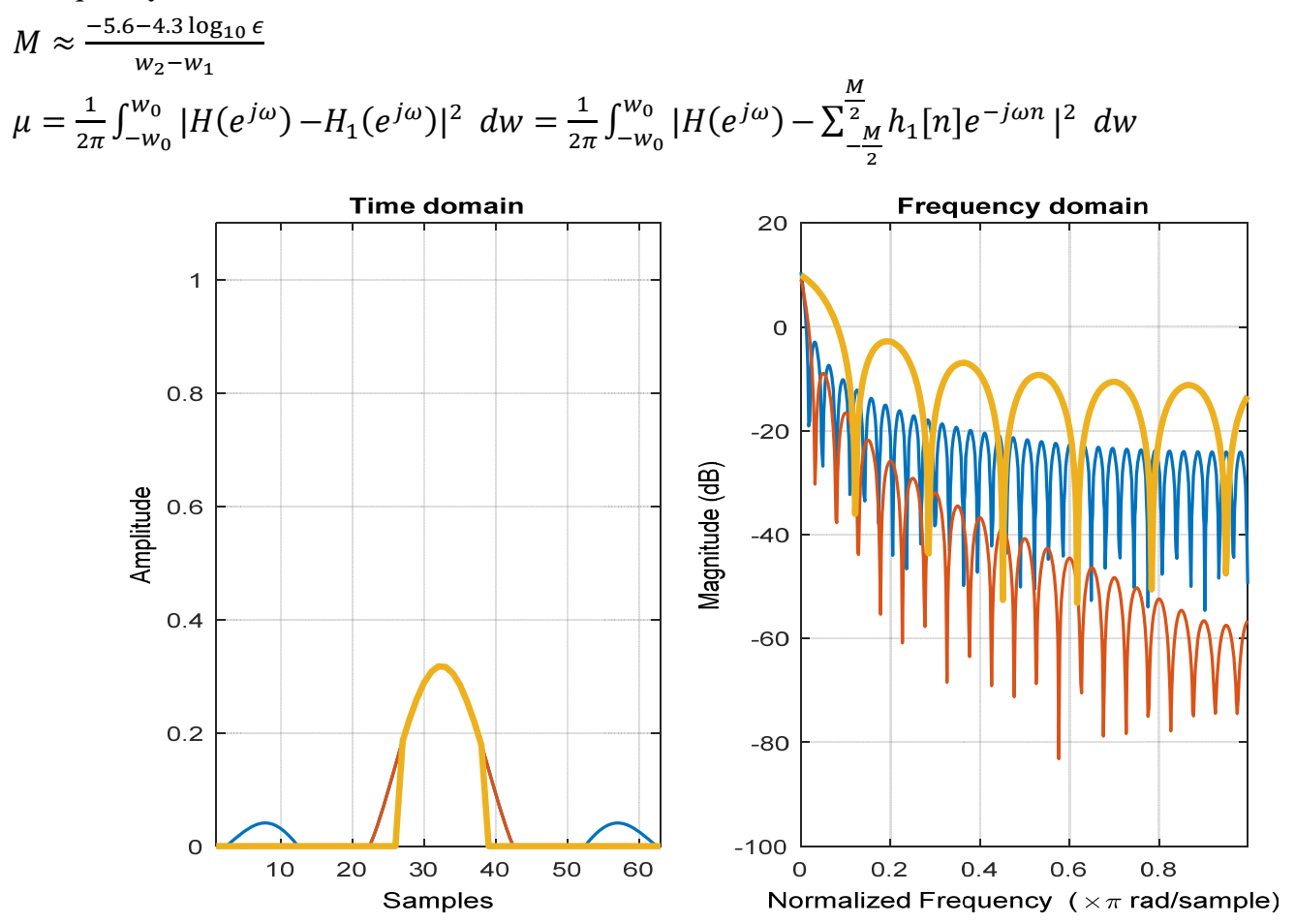

Fig. 3.Behavior of the filter output in time and frequency output.

When performing the convolution, the coefficients of the transfer function are obtained in ascending order. These coefficients accompany the independent variable by expanding the summative term an amount of times $\mathrm{M}$. For example, when $\mathrm{M}$ is equal to 2 and the cutoff frequency is $6 \mathrm{kHz}$, the expression observed in equation 15 is obtained.

$$
H(z)=0.3214689087634+0.3570621824733 z^{-1}+0.3214689087634 z^{-2}
$$

There are some support tools to design FIR filters, such as MATLAB's FDATOOL, which estimates the coefficients applying the methodology described as shown in Fig. 4.

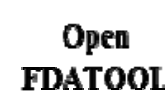

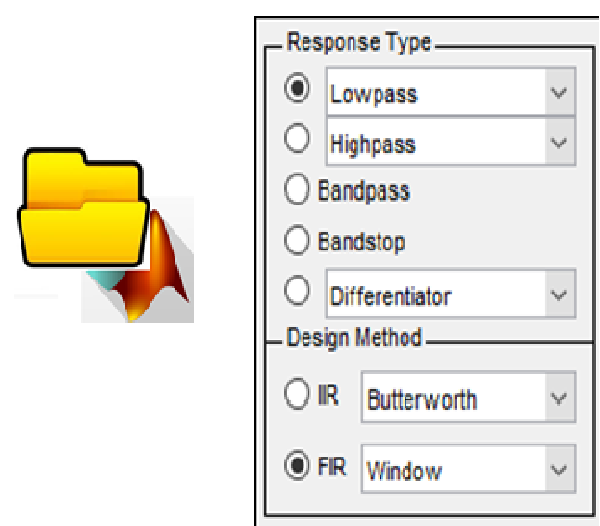

Set Design

Criteria

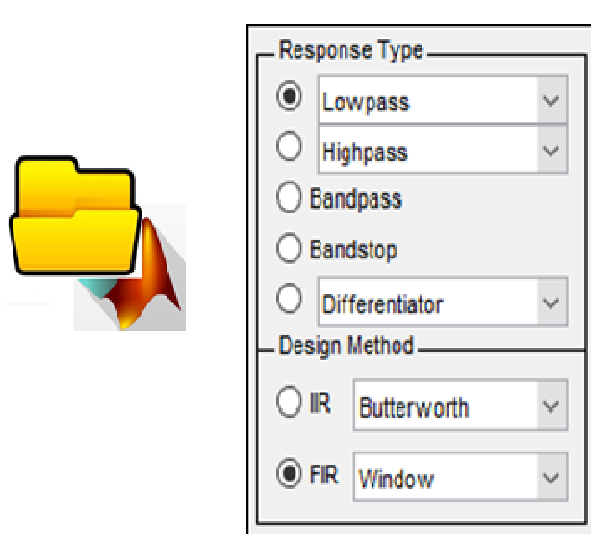

\section{Select Cutoff E requencies,} Orler and Type

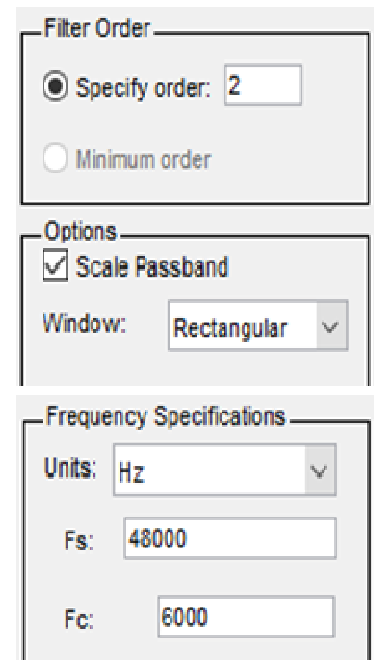

\section{Generate Coefficients}

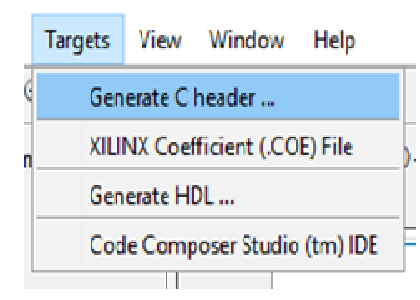

Fig. 4. Steps to generate FIR coefficients using MATLAB 
The coefficients are generated in a plain text file, which is incorporated into the controller through a routine (as shown in Algorithm 1). This routine is responsible for taking samples of the signal to be filtered using the digital analog converter of the controller with the frequency established during the design. When sampling the signal, each sample is stored inside a memory, whose quantity is determined by the order of the filter and each of them is multiplied by the coefficients mentioned above.

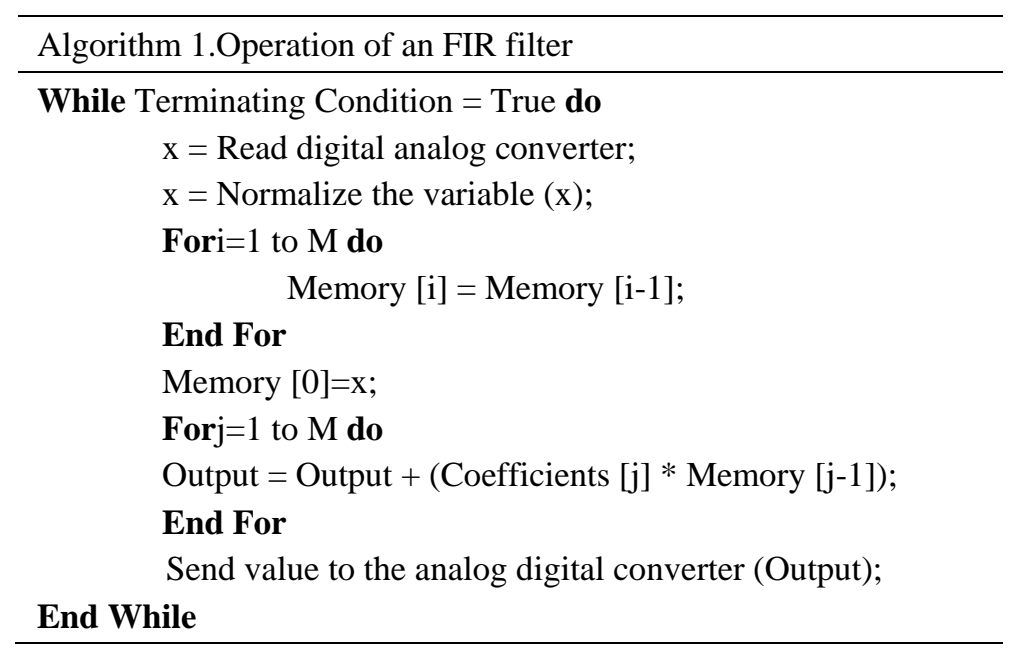

Fig. 5 shows the architecture used in the microcontroller to perform the filter function. As seen there is an analog to digital converter and its complement that are responsible for filtering and modulating the input and output information. The PWM function is responsible for establishing the sampling time used by the converters to reconstruct the signal.

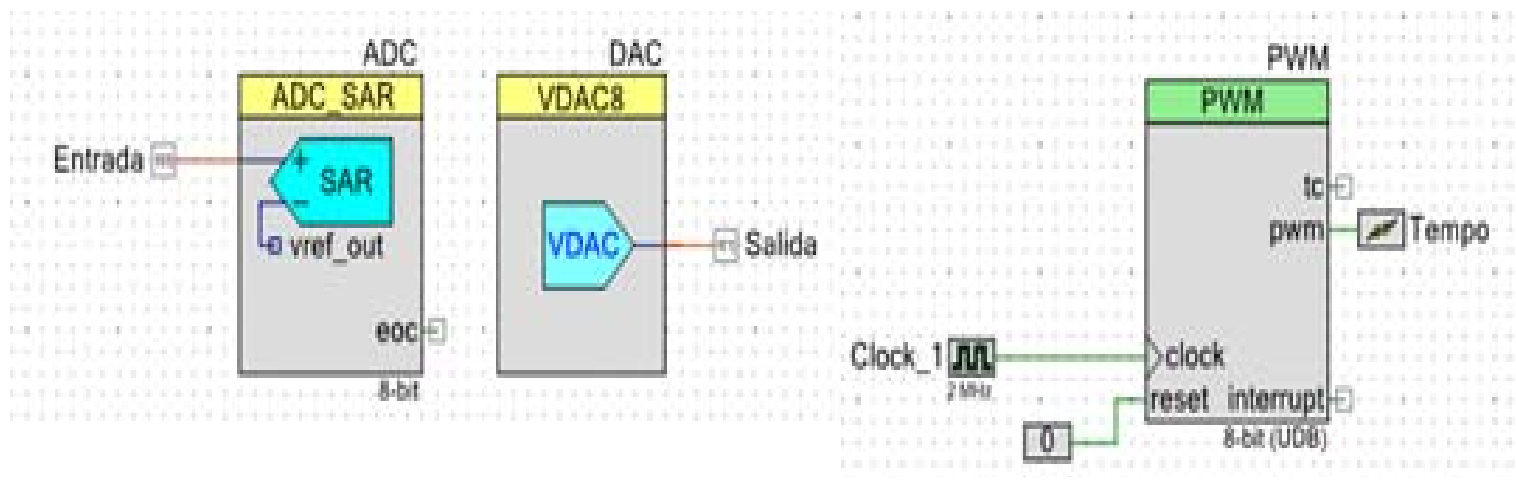

Fig. 5. Architecture incorporated in the PSOC 5LP.

\section{EXPERIMENTAL RESULTS}

The methodology shown to implement a FIR filter in PSOC 5LP was tested by implementing several filter schemes. The coefficients of the low-pass, high-pass, band-pass and eliminated-band filters were determined, whose resulting transfer function was simulated before being implemented in the controller and the results obtained are shown in Tables I and II. To measure the results, an oscilloscope was connected to the output of a signal generator, which is connected in parallel to the input of the microcontroller and the output of the controller is connected to another channel of the oscilloscope.

The low-pass and high-pass filters were designed at a cutoff frequency of $4 \mathrm{KHz}$ and the signal is sampled at $48 \mathrm{KHz}$. Band-pass and band-eliminated filters were designed at cutoff frequencies at $2 \mathrm{KHz}$ and $6 \mathrm{KHz}$. The test signal varies in frequency from 2 to $4 \mathrm{KHz}$ and has an amplitude of $1 \mathrm{~V}$. 
TABLEI. Results obtained when implementing a low-pass and high-pass filter

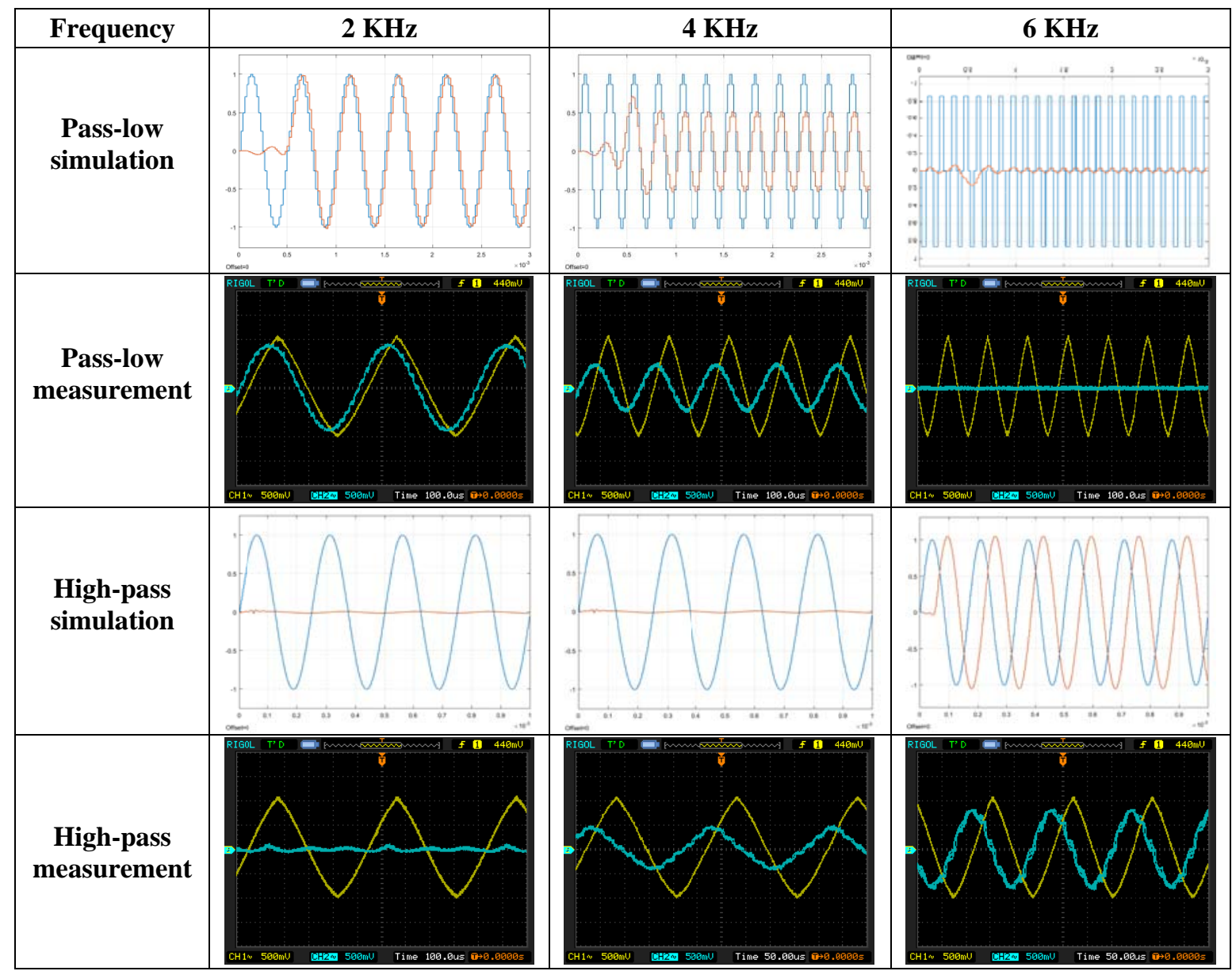

TABLE II. Results obtained when implementing a band-pass and band-pass filter

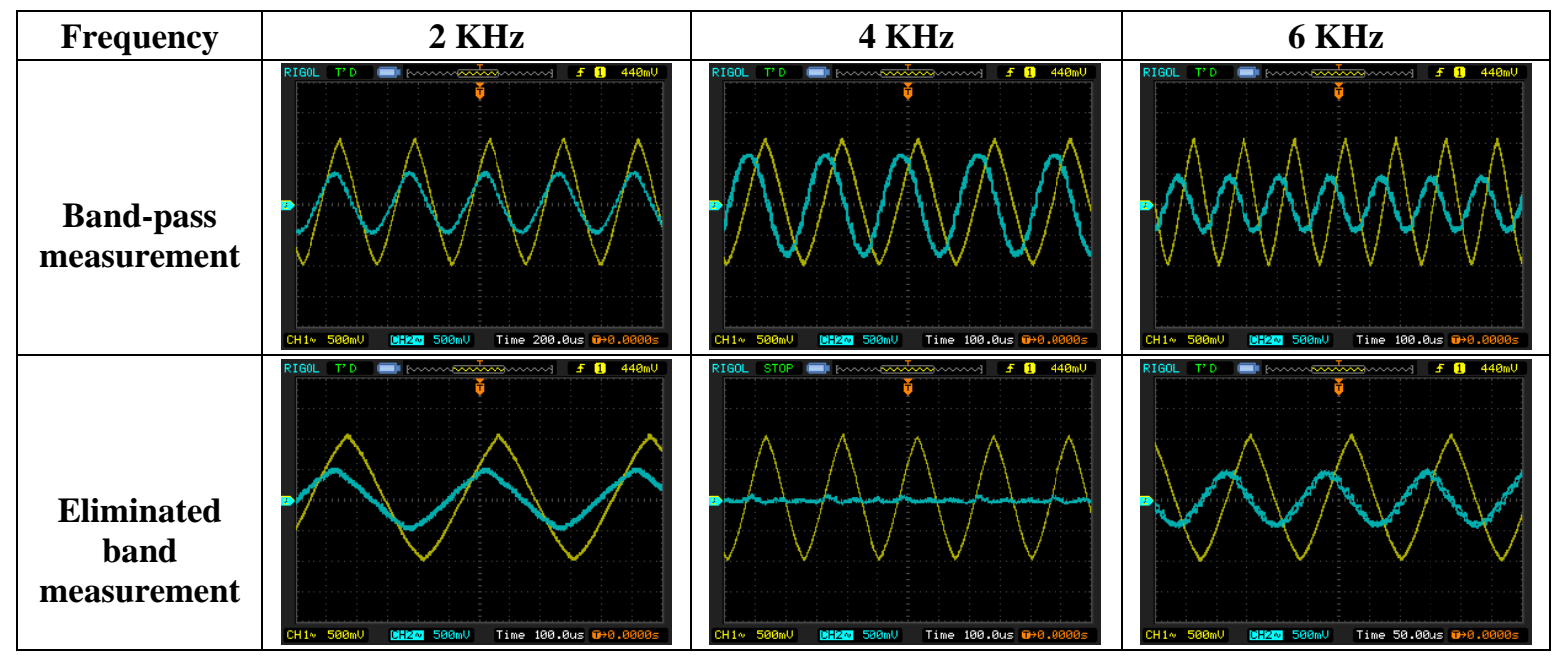

\section{CONCLUSIONS}

The FIR filter design methodology allows to implement different kinds of filters in the frequency domain, among which are: pass-low, pass-high, pass-band and band-eliminated. As shown in Tables I and II, the filters implemented work well around the cutoff frequencies, however, a greater attenuation of the frequencies eliminated in the simulation can be observed, this is due to the fact that the coefficients are truncated at the moment of implement the transfer function in the controller.

The implementation of the FIR filter in the PSOC 5LP controller works correctly up to frequencies of 12 $\mathrm{KHz}$, as shown in Figure 6 as the frequency of the input signal increases, the controller reduces its ability to process the signal correctly, since the controller does not offer a higher sampling frequency to improve the characteristics of the input signal. 
The order of the filters implemented is 50 for low-pass and high-pass filters and 100 for band-pass and bandelimination filters, since when performing experimental tests with a lower order the filter did not work properly. In such a way, this was the lowest order obtained with the strategy proposed in this paper, to design FIR architecture filters.

\section{ACKNOWLEDGMENT}

This work was supported by the Universidad Distrital Francisco José de Caldas Technological Faculty. The views expressed in this paper are not necessarily endorsed by District University. The authors thank the research group ARMOS for the evaluation carried out on prototypes of ideas and strategies

\section{REFERENCES}

[1] H. Almahzumy, F. Ardilla and B. S. Marta, "Board PSoC library design and evaluation for mobile robot implementation," 2017 International Electronics Symposium on Engineering Technology and Applications (IES-ETA), Surabaya, 2017, pp. 257-262. doi: 10.1109/ELECSYM.2017.8240413

[2] R. J. M. Mercado, "Design of wireless sensor networks using embedded Programmable System-on-Chip (PSoC) as applied to community-based flood early warning systems (CBFEWS)," 2016 International Conference on Advances in Electrical, Electronic and Systems Engineering (ICAEES), Putrajaya, 2016, pp. 214-223. doi: 10.1109/ICAEES.2016.7888041

[3] S. Gharavi, A. Ramezani and J. Frounchi, "Implementation of a PSoC-based flexible and low-power processor," 2016 24th Iranian Conference on Electrical Engineering (ICEE), Shiraz, 2016, pp. 1780-1784. doi: 10.1109/IranianCEE.2016.7585809

[4] K. Chengeta and S. Viriri, "A survey on facial recognition based on local directional and local binary patterns," 2018 Conference on Information Communications Technology and Society (ICTAS), Durban, South Africa, 2018, pp. 1-6.

[5] C. Y. Peng and R. C. Chen, "Voice recognition by Google Home and Raspberry Pi for smart socket control," 2018 Tenth International Conference on Advanced Computational Intelligence (ICACI), Xiamen, China, 2018, pp. 324-329. doi: 10.1109/ICACI.2018.8377477

[6] W. H. Lee, Y. T. Lee, J. W. Kim, H. Takao, K. Sawada and M. Ishida, "Wireless smart temperature sensor using pulse width modulation method," 2007 70th ARFTG Microwave Measurement Conference (ARFTG), Tempe, AZ, USA, 2007, pp. 1-4.doi: 10.1109/ARFTG.2007.8376233

[7] D. L. Kuhite and M. S. Madankar, "Wireless audio transmission system for real-time applications — A review," 2017 International Conference on Inventive Systems and Control (ICISC), Coimbatore, 2017, pp. 1-5. doi: 10.1109/ICISC.2017.8068680

[8] Z. Akhtar, G. Kumar, S. Bakshi and H. Proenca, "Experiments with Ocular Biometric Datasets: A Practitioner's Guideline," in IT Professional, vol. 20, no. 3, pp. 50-63, May/Jun. 2018. doi: 10.1109/MITP.2018.032501748

[9] C. Zeintl and H. G. Brachtendorf, "Linear phase design of lattice wave digital filters," 2018 28th International Conference Radioelektronika (RADIOELEKTRONIKA), Prague, Czech Republic, 2018, pp. 1-5. doi: 10.1109/RADIOELEK.2018.8376376

[10] H. Park, M. Yu, Y. Jung and S. Lee, "Design of Reconfigurable Digital IF Filter with Low Complexity," in IEEE Transactions on Circuits and Systems II: Express Briefs. doi: 10.1109/TCSII.2018.2844283

[11] M. B. Trimale and Chilveri, "A review: FIR filter implementation," 2017 2nd IEEE International Conference on Recent Trends in Electronics, Information \& Communication Technology (RTEICT), Bangalore, 2017, pp. 137-141. doi: 10.1109/RTEICT.2017.8256573

[12] F. B. Elissa and M. J. Mismar, "Adaptive FIR filter for frequency estimation of sinusoids using particle swarm optimization," 2016 5th International Conference on Electronic Devices, Systems and Applications (ICEDSA), Ras Al Khaimah, 2016, pp. 1-4. doi: 10.1109/ICEDSA.2016.7818529

[13] S. R. Sutradhar, N. Sayadat, A. Rahman, S. Munira, A. K. M. F. Haque and S. N. Sakib, "IIR based digital filter design and performance analysis," 2017 2nd International Conference on Telecommunication and Networks (TEL-NET), Noida, 2017, pp. 1-6. doi: 10.1109/TEL-NET.2017.8343596

[14] S. Song, S. X. Chew, X. Yi, L. Nguyen and R. A. Minasian, "Tunable Single Passband Microwave Photonic Filter based on Integrated Optical Double Notch Filter," in Journal of Lightwave Technology. doi: 10.1109/JLT.2018.2841647

[15] M. G. Kulkarni, A. N. Cheeran, K. P. Ray and S. S. Kakatkar, "Design and implementation of CPW low pass filter with good filter selectivity and sharpness factor," 2018 3rd International Conference on Microwave and Photonics (ICMAP), Dhanbad, 2018, pp. 1-2. doi: 10.1109/ICMAP.2018.8354617

[16] L. Burgnies et al., "High-Pass Sub-mmWave Filtering by Woven Textile Metamaterials," in IEEE Transactions on Terahertz Science and Technology. doi: 10.1109/TTHZ.2018.2844096

[17] S. O. Gilani, Y. Ilyas and M. Jamil, "Power line noise removal from ECG signal using notch, band stop and adaptive filters," 2018 International Conference on Electronics, Information, and Communication (ICEIC), Honolulu, HI, 2018, pp. 1-4. doi: 10.23919/ELINFOCOM.2018.8330569 